\title{
Three-Dimensional Self-Organization in Nanocomposite Layered Systems by Ultrafast Laser Pulses
}

\author{
Zeming Liu' ${ }^{1}$, Jan Siegel2*, Mario Garcia-Lechuga ${ }^{2}$, Thierry Epicier ${ }^{3}$, Yaya \\ Lefkir $^{1}$, Stéphanie Reynaud ${ }^{1}$, Matthieu Bugnet ${ }^{3}$, Francis Vocanson ${ }^{1}$, Javier \\ Solis ${ }^{2}$, Guy Vitrant ${ }^{4}$, Nathalie Destouches ${ }^{1 *}$ \\ ${ }^{1}$ Univ Lyon, UJM-Saint-Etienne, CNRS, Institut d Optique Graduate School, Laboratoire Hubert \\ Curien UMR 5516, F-42023, SAINT-ETIENNE, France, \\ ${ }^{2}$ Laser Processing Group, Instituto de Optica, Serrano 121, 28006 Madrid, Spain \\ ${ }^{3}$ MATEIS, umr CNRS 5510, University of Lyon, INSA Lyon, University Lyon I, Villeurbanne, \\ FRANCE \\ ${ }^{4}$ IMEP-LAHC, Minatec, Grenoble-INP, CNRS-UMR 5130, F-38016 Grenoble, France \\ *email: nathalie.destouches@univ-st-etienne.fr; j.siegel@io.cfmac.csic.es
}

\begin{abstract}
Controlling plasmonic systems with nanometre resolution in transparent films and their colors over large non-planar areas is a key issue for spreading their use in various industrial fields. Using light to direct self-organization mechanisms provides high-speed and flexible processes to meet this challenge. Here, we describe a route for the laser-induced self-organization of metallic nanostructures in 3D. Going beyond the production of planar nanopatterns, we demonstrate that ultrafast laser-induced excitation combined with non-linear feedback mechanisms in a nanocomposite thin film can lead to 3D self-organized nanostructured films. The process, which can be extended to complex layered composite systems, produces highly uniform large-area nanopatterns. We show that 3D self-organization originates from the simultaneous excitation of independent optical modes at different depths in the film and is activated by the plasmon-induced charge separation and thermally-induced NP growth mechanisms. This laser color marking technique enables multiplexed optical image encoding

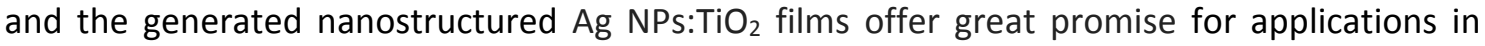
solar energy harvesting, photocatalysis or photochromic devices.
\end{abstract}

\section{Keywords}

Ultrafast photonics; Laser-induced self-organization; Nanostructured thin film; plasmonic nanomaterials; plasmonic colors

Controlling metallic nanostructures over large non-planar areas with high flexibility and speed is of strategic importance for developing industrial applications of plasmonic systems. ${ }^{1,2}$ Electron-beam lithography has been used recently to demonstrate innovative applications of plasmonic colors for active color displays, data storage or polarization microscopy, ${ }^{3-5}$ but this technique is prohibitive in terms of time and cost. Other techniques as optical interference lithography, nanoimprint ${ }^{6}$ or directed-assembly ${ }^{7-9}$ are more appropriate for producing largescale nanostructured materials, but they lack versatility. In contrast, laser-based techniques combine all the benefits sought for industrial developments and offer two alternative

ACS Nano, 2017, 11 (5), pp 5031-5040, DOI: 10.1021/acsnano.7b01748

Publication Date (Web): May 4, 2017, Copyright (C 2017 American Chemical Society 
strategies. The first one is based on controlling the shape anisotropy of NPs and exploits the high peak intensity of femtosecond ( $f s$ ) laser pulses or the thermal component of nanosecond (ns) pulses to change the NP shape. ${ }^{3,4,10-14}$ The second approach, even more promising, is based on self-organization processes and controls simultaneously the intrinsic plasmonic response of individual NPs and their near- and far-field coupling effects. Using this approach, a few papers have reported ultrafast laser-induced melting and merging of metallic NPs at surfaces to form regular gratings with ${ }^{15}$ or without ${ }^{16,17} \mathrm{DC}$ electric field assistance. Addressing the challenge to modify NPs inside a dielectric matrix, Loeschner and co-workers have reported the generation of gold NP gratings embedded in polymer films using fs laser pulses, ${ }^{18}$ while our group triggered the self-organized growth of silver NPs in high-index $\mathrm{TiO}_{2}$ films with continuous wave (cw) laser light. ${ }^{19}$

A common feature of all these self-organized metallic nanostructures is that their period results from the interference of the incident laser wave with optical modes excited in the structures. Interestingly, the dependence of the grating orientation on the laser polarization is not universal, but is found to be either parallel $\left.\right|^{18,19}$ or perpendicular. ${ }^{15,17}$ This fact implies the presence of different types of optical modes as the origin of the interference pattern, depending on the system. Quasi-guided modes ${ }^{19}$ and surface waves ${ }^{17}$ have been identified to be involved in such self-organization mechanisms. However, all NP gratings reported so far have been produced in single planes, buried below or lying at the material surface, as a result of interferences between the incident wave and a single optical mode. This applies both to NP gratings and planar NP grid structures. ${ }^{17,18,20}$

Here, we show that 3D self-organization can be activated and controlled in nanocomposite films by fs laser irradiation, resulting in plasmonic nanostructured films with highly regular periodic lattices located at different depths. The paper demonstrates that this 3D self-organization mechanism finds its origin in the simultaneous excitation of two orthogonal optical modes. It also explains how plasmon-induced charge transfers and thermally-induced growth mechanisms that follow absorption of the incident photons by $\mathrm{Ag}$ NPs trigger the processes. Emphasizing the fact that the nanopattern size can be extended indefinitely with high-speed scanning, we finally demonstrate the high potential of this nanostructuring strategy for color marking and multiplexed optical image encoding.

\section{RESULTS AND DISCUSSION}

The system under study is composed (Figure 1a) of a glass substrate covered with a mesoporous layer of amorphous $\mathrm{TiO}_{2}$ loaded with silver salt and small silver NPs (see methods). ${ }^{19,21}$ Self-organized 3D nanostructures can be generated in a certain range of laser fluence and scan speed (see methods) using a high repetition rate fs laser (500 kHz) exciting the localized surface plasmon resonance (LSPR) of Ag NPs. A localized surface plasmon is the result of the confinement of a surface plasmon in a nanoparticle that is smaller than the light wavelength used to excite the plasmon. As shown hereafter, their post-mortem characterization informs clearly about the nature of the optical phenomena at their origin. However, the paper intends to demonstrate the fundamental role of temperature in triggering $3 D$ self-organization rather than $2 \mathrm{D}$ and, for this, compare $2 \mathrm{D}$ and $3 \mathrm{D}$ nanostructures, obtained by changing only the scanning speed.

ACS Nano, 2017, 11 (5), pp 5031-5040, DOI: 10.1021/acsnano.7b01748

Publication Date (Web): May 4, 2017, Copyright (C) 2017 American Chemical Society 

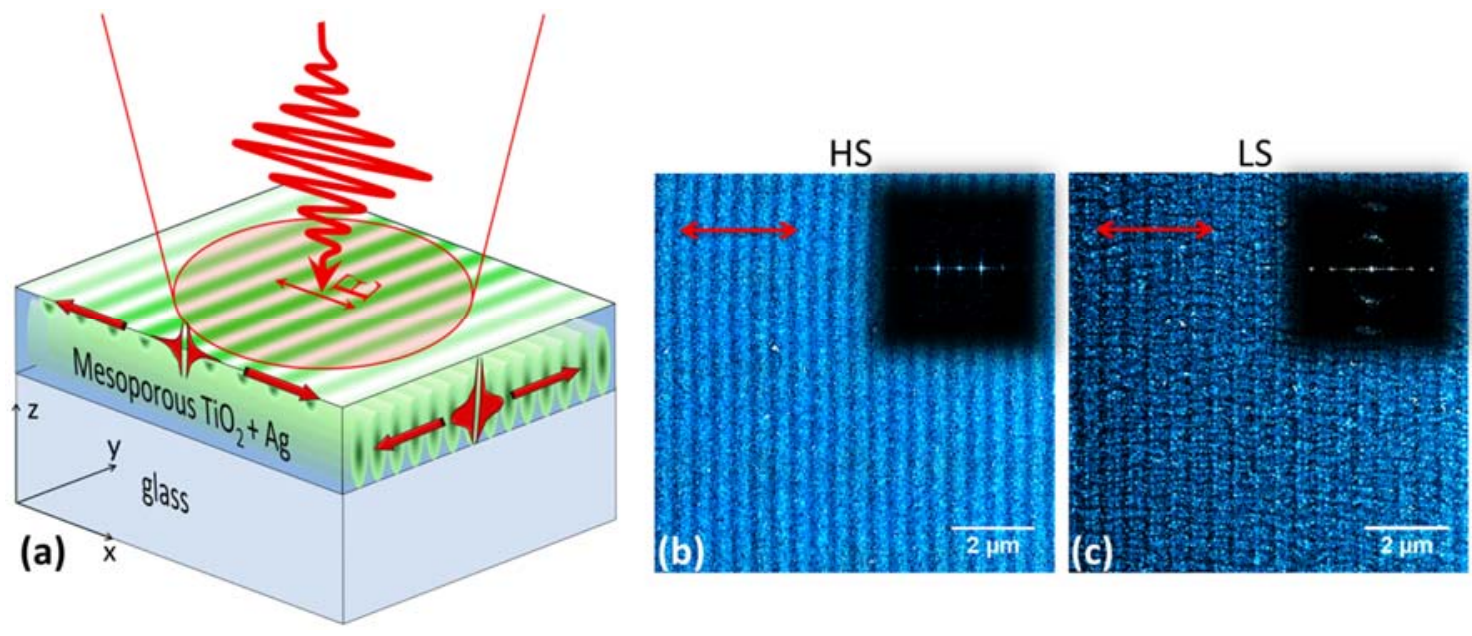

Figure 1: Overview of directed 3D self-organization. (a) Layout of the optical phenomena at the origin of 3D self-organization in the nanocomposite film. The linearly polarized incident light excites simultaneously a surface wave and a guided wave propagating in perpendicular directions. Both waves interfere with incident light and give rise to an intensity modulation either near the top surface of the film along the polarization direction or within the film in the perpendicular direction. The two periods are different. (b-c) SEM micrographs of structures HS (b) and LS (c) and their respective FFT images (inset). These two nanostructured $\mathrm{Ag}: \mathrm{TiO}_{2}$ films were irradiated with a constant scanning speed of 100 $\mathrm{mm} \cdot \mathrm{s}^{-1}$ (b) or $10 \mathrm{~mm} \cdot \mathrm{s}^{-1}$ (c) using a linearly polarized laser beam whose polarization direction is given by the red double arrow.

Scanning electron microscopy (SEM) images of these self-organized nanostructures are shown in Figure $1 \mathrm{~b}-\mathrm{c}$. Both samples were irradiated at $515 \mathrm{~nm}$ wavelength with a weakly focused beam ( $33 \mu \mathrm{m}$ spot diameter at $1 / \mathrm{e}^{2}$ ) scanning the sample surface at two different speeds of $100 \mathrm{~mm} \cdot \mathrm{s}^{-1}$ or $10 \mathrm{~mm} \cdot \mathrm{s}^{-1}$, which give rise to structures named HS (for high speed) and LS (for low speed), respectively. Both of them exhibit highly regular self-organized patterns that were extended over areas of several $\mathrm{cm}^{2}$. Although the first observation of laser-induced self-organized nanostructures also known as LIPSS (Laser Induced Periodic Surface Structures) dates back to $1965,{ }^{22}$ such a high uniformity and long-range order on planar structures was highlighted only few years ago, paving the way for real-world applications. ${ }^{23}$ The high uniformity was achieved here by optimizing scan parameters (see method).

\section{D self-organized nanocomposite film.}

Structure HS (Fig. 1b) exhibits a periodic line pattern perpendicular to the incident laser polarization with a period of $490 \mathrm{~nm} \pm 5 \mathrm{~nm}$. A high-magnification and in-depth inspection of this structure by high angle annular dark field (HAADF) scanning transmission electron microscopy (STEM) reveals the presence of LIPSS with height amplitude of around $40 \mathrm{~nm}$ and Ag NPs up to $20 \mathrm{~nm}$ in diameter periodically distributed in the crescent-shape region below the grooves (see Fig. 2 and Supplementary Information). The 4D STEM analysis (see methods) of Figure $2 \mathrm{~d}$ also shows that the film is denser in this region due to the collapse of the initial mesostructure (see also Supplementary Information) and a partial crystallization of $\mathrm{TiO}_{2}$ in the close vicinity of the grown Ag NPs. 

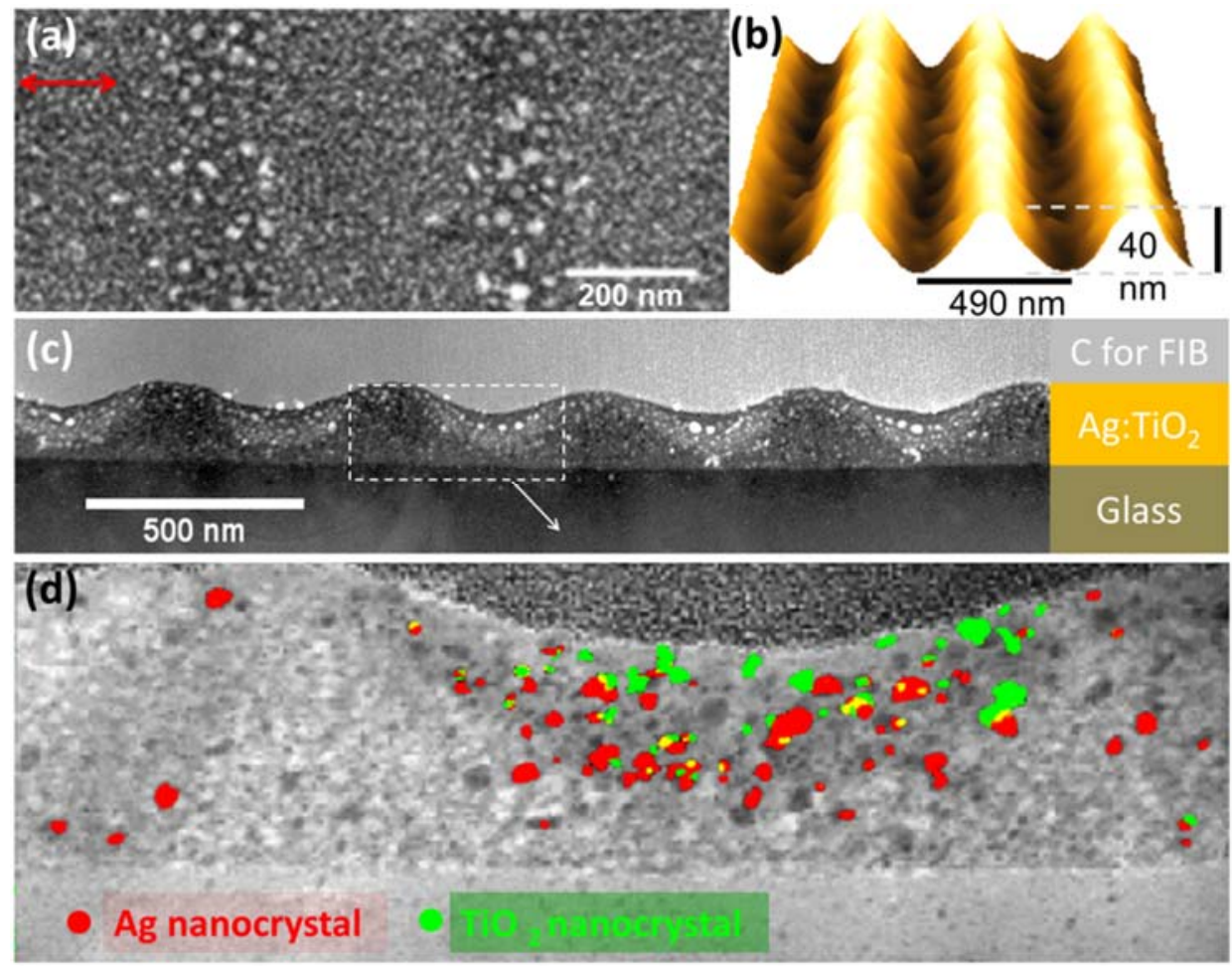

Figure 2: HS nanostructure. (a) Top view HAADF STEM micrograph with a red double arrow indicating the laser polarization. (b) Atomic force microscopy (AFM) topography. (c) Low magnification HAADFSTEM image of the film cross-section showing the LIPSS and the typical region of interest (white dashed frame) characterized by '4D-STEM' whose final result is given in (d). (d) Bright field reconstructed STEM image, in which dark areas are diffracting crystalline regions. Red and green colors have been used to label areas that have been positively identified as crystallized $\mathrm{Ag}$ and $\mathrm{TiO}_{2} \mathrm{NPs}$, respectively. The indepth superposition of $\mathrm{Ag}$ and $\mathrm{TiO}_{2}$ nanocrystals gives the yellow color.

\section{D self-organized nanocomposite film.}

Structure LS (Fig. 1c) features two superimposed orthogonal line patterns whose periods are $310 \mathrm{~nm} \pm 5 \mathrm{~nm}$ (for lines parallel to the laser polarization) and $500 \mathrm{~nm} \pm 5 \mathrm{~nm}$ (in the perpendicular direction). These two self-organized patterns are located at two different depths in the sample. AFM shows a single periodic corrugation of the sample topography (Fig. 3a) whose lines are perpendicular to the laser polarization. Instead, lines parallel to the laser polarization are only revealed by HAADF STEM characterizations and correspond to buried $\mathrm{Ag}$ NPs (Figure 3b-c; see also Supplementary Information). Unlike in structure HS, in the LS structure Ag NPs align parallel to the laser polarization, independently from the LIPSS, to form a grating whose period is $\sim 310 \mathrm{~nm}$ and which is now located very close to the film-substrate interface (Fig. 3c). Ag NPs grow, with an oblate shape, up to larger sizes with a broad size distribution typically from 20 to $100 \mathrm{~nm}$ (Supplementary Information), to arrange in lines about $195 \mathrm{~nm}$ wide. All silver has diffused towards the substrate, in strong contrast to the HS structure, and the film is now denser. 

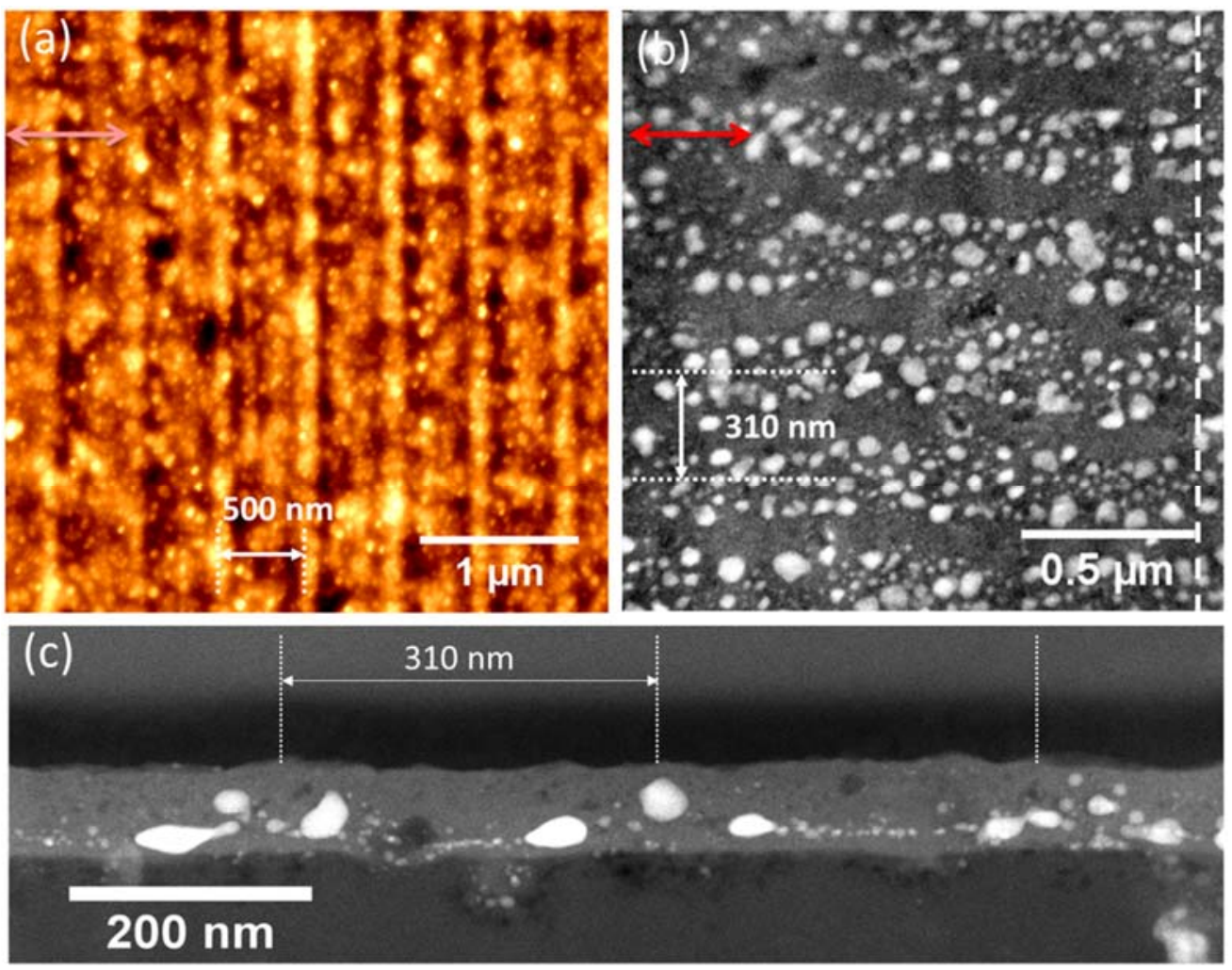

Figure 3: LS nanostructures. (a) Surface topography measured by AFM and (b) in-depth top view of the film nanostructure imaged by HAADF STEM. The double red arrow shows the direction of the laser polarization in both images. (c) Typical HAADF STEM micrograph of the sample cross-section (FIB preparation extracted parallel to the white dashed line in (b)).

\section{Optical mechanisms involved in the formation of the NP gratings.}

The main difference between structures HS and LS is that Ag NPs grow in the grooves of LIPSS in HS whereas they form a different self-organized pattern, buried in the film and independent from LIPSS, in LS. The second case nicely demonstrates that two independent propagating modes can be excited simultaneously in orthogonal directions at different depths in the nanocomposite film, as sketched in Figure 1a. They correspond to a surface mode and a guided mode, respectively. Individually, either of them has already been shown to be capable of triggering self-organized patterns in any of the two directions, ${ }^{15,17-19}$ with periods (with respect to the laser wavelength) similar to the ones reported here, but never simultaneously in the same system before, leading only to $2 \mathrm{D}$ self-organized patterns. These modes are excited through scattering at heterogeneities and are initially no more than a perturbation signal generated by randomly distributed scatterers, preventing phase matching conditions and an efficient coupling of light in the guiding film. However, each of these modes interferes with the incident wave and gives rise to a modulated intensity in the plane where it propagates. The optical interference pattern triggers the grating formation. The grating then creates a positive feedback loop by enhancing the coupling yield of the incident wave to the mode, i.e. increasing the contrast of the interference pattern and finally promoting the grating formation itself. ${ }^{19,23}$ Whatever the nature of the mode, and provided that the incident wave is under normal incidence, the period $\Lambda$ of the interference pattern that is also the grating period can be written as: $\Lambda=\frac{\lambda}{\operatorname{Re}(\eta)}$, where $\lambda$ is the laser wavelength and $\operatorname{Re}(\eta)$ the real part of the effective refractive index of the considered mode, which can be a surface or guided mode. ${ }^{24}$ 
Let us first consider the surface mode, which has often been identified as a surface plasmon polariton no matter if the surface is metal, semiconductor, or dielectric due to the transient metal-like state that can be achieved by the high peak intensity of ultrashort laser pulses, creating a high density of free electrons. ${ }^{25-29}$ However, in the present case we deal with a composite film in which only small Ag NPs are directly excited at resonance. It is therefore useful to emphasize the fact that the effective dielectric constant of the film top surface is tuned towards that of a metal during the pulse duration thanks to the high density of Ag NPs and their ability to transfer charges to the conduction band of the $\mathrm{TiO}_{2}$ matrix (see the next sub-section). The transverse magnetic nature of the surface plasmon can then explain that LIPSS form perpendicular to the incident laser polarization. In this case $\eta$ can be expressed as the effective index of the surface plasmon mode, which is close to 1 for metals, ${ }^{28}$ and fully consistent with the period of LIPSS observed in structure HS.

Let us now consider the guided mode, which leads to the formation of the buried NP grating of structure LS. Importantly, its period with respect to the laser wavelength and its orientation with respect to the laser polarization is comparable to the one we had previously reported when creating self-organized NP gratings with $\mathrm{cw}$ lasers buried in the same mesoporous $\mathrm{TiO}_{2}$ films. ${ }^{19}$ The presence of a guided mode was then demonstrated through spectral measurements that are less obvious to interpret in the present case due to the presence of two stacked perpendicular nanopatterns (see sub-section "Origin of dichroic colors" for spectral characterizations). We can, however, propose the same origin for the generation of the LS grating structure. Light initially scattered by heterogeneities embedded inside the film (that are mainly the growing Ag NPs due to their high dielectric contrast in the matrix) is minimal in the direction of the incident electric field and maximal in the direction perpendicular, especially if we consider a dipolar approximation that works well for scatterers much smaller than the laser wavelength. The scattered field is therefore mainly coupled into the guided mode propagating perpendicular to the laser polarization. This leads to an interference pattern of propagated light with directly incident light, with lines of higher intensity that are parallel to the laser polarization. The positive feedback created by the growing grating only works for light that is efficiently coupled into the guided mode in the direction perpendicular to the grating lines. This leads to the generation of a buried NP grating whose lines are parallel to the laser polarization.

While optical phenomena are crucial to understand the origin of self-organization as demonstrated above, other mechanisms need to be considered to explain the growth of the NPs and the transient change in the matrix refractive index. Figure 4 shows a simplified scheme of the processes involved, as discussed next.

\section{Short timescale: Charge transfer from Ag NPs to the $\mathrm{TiO}_{2}$ conduction band and NP heating.}

The absorption of the incident light by the NPs generates a high density of excited electrons through the LSPR. During a very short timescale, electron-electron scattering gives rise to hot electrons that are not in thermodynamic equilibrium with the Ag lattice before typically few hundreds of $\mathrm{fs}$. These non-equilibrium electrons penetrate into the material with ballistic velocities of the order of $10^{6} \mathrm{~m} / \mathrm{s},{ }^{30}$ and their energy range is estimated between $1 \mathrm{eV}$ and $4 \mathrm{eV}$ for Ag NPs. ${ }^{31}$ Those electrons whose kinetic energy overcomes the Schottky barrier that forms at the metal nanoparticles $/ \mathrm{TiO}_{2}$ interface and that can be as low as $1 \mathrm{eV}$ in such systems, can be injected into the $\mathrm{TiO}_{2}$, as shown in Fig. 4a. ${ }^{32,33}$ This plasmon-induced charge transfer has already been measured to occur in less than $240 \mathrm{fs}^{34}$ Added to the possible near-field mediated multiphoton excitation of $\mathrm{TiO}_{2}$ (see "laser-induced temperature rise" in Supplementary Information), the strong increase of the free electron density in $\mathrm{TiO}_{2}$ (Fig. 4a-b)

ACS Nano, 2017, 11 (5), pp 5031-5040, DOI: 10.1021/acsnano.7b01748

Publication Date (Web): May 4, 2017, Copyright (C 2017 American Chemical Society 
triggers the transient metallic behavior of the film required for the excitation of surface plasmon polaritons at the film-air interface, as described in the previous section.

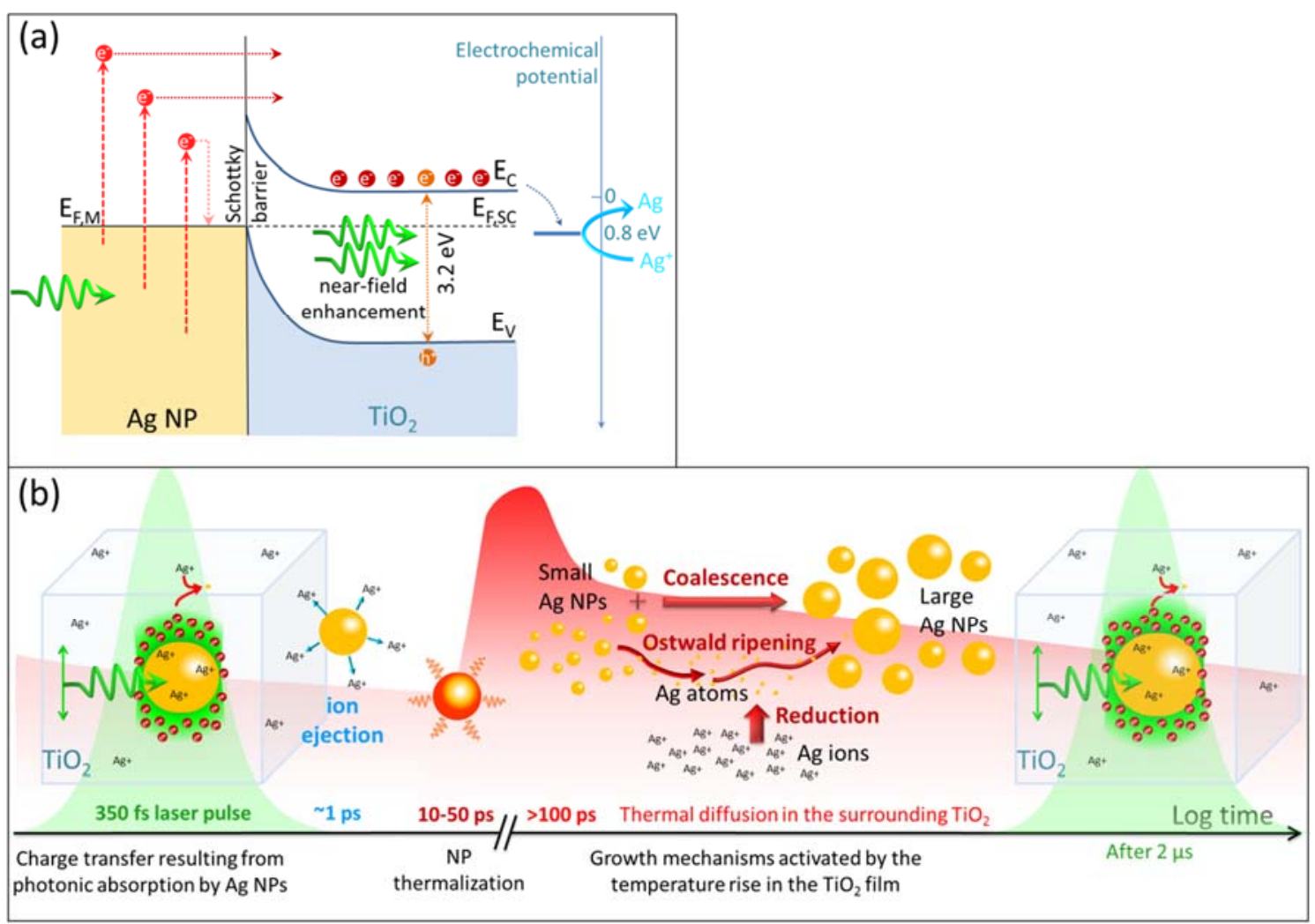

Figure 4: Mechanisms involved upon the interaction of a high-repetition rate fs laser pulse train with a nanocomposite film. (a) Energetic diagram of the $\mathrm{Ag} \mathrm{NPs}$ and the $\mathrm{TiO}_{2}$ matrix at their interface relative to the redox potential of $\mathrm{Ag}^{+} / \mathrm{Ag}$. Hot electrons with high energy can surpass the Schottky barrier and migrate to the conduction band of $\mathrm{TiO}_{2}$. Electron-hole pairs can also be generated in the semiconductor matrix by two-photon absorption, which is only expected to occur in the near-field of Ag NPs. Excited electrons can then reduce $\mathrm{Ag}^{+}$ions present in the mesoporous $\mathrm{TiO}_{2}$ matrix thanks to a redox potential located just above the $\mathrm{TiO}_{2}$ conduction band energy. (b) Incident photons are mainly absorbed by $\mathrm{Ag}$ NPs whose LSPR relaxes though the generation of hot electrons that can migrate to the conduction band of $\mathrm{TiO}_{2}$. The LSPR also creates a strong near-field enhancement along the incident polarization (double arrow), which enables multiphoton absorption by $\mathrm{TiO}_{2}$. Charge transfer and charge generation create a high density of charges in the $\mathrm{TiO}_{2}$ matrix. Ion ejection from Ag NPs can occur followed by NP thermalization, which heats the matrix through thermal diffusion. A high temperature rise in the matrix (red background) enables the growth mechanisms that can lead to large Ag NPs if the time frame is long enough.

The massive electron transfer through the Schottky barrier leaves behind positively charged Ag NPs, leading to strong electrostatic repulsion forces between ions. This can cause the breakup of atomic bonds and the ejection of charged $\mathrm{Ag}^{+}$atoms over a time scale of the order of $1 \mathrm{ps}$, according to a process that is reported as Coulomb explosion in papers dealing

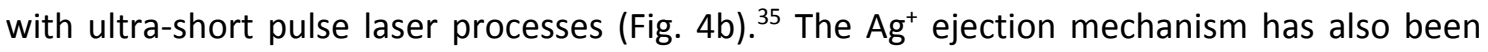
observed in $\mathrm{TiO}_{2}$ films even upon low intensity cw visible laser exposure (due to the low Schottky barrier) and led to the anisotropic reshaping or the disappearance of Ag NPs. ${ }^{36,37}$ It has to be emphasized that this process is leading to NP shrinking and thus opposed to the temperature-induced growth described hereafter. Although $\mathrm{Ag}^{+}$ion ejection certainly occurs in the present experiments, it seems to be compensated and overtaken by the growth mechanisms since only larger isotropic NPs are finally observed.

ACS Nano, 2017, 11 (5), pp 5031-5040, DOI: 10.1021/acsnano.7b01748

Publication Date (Web): May 4, 2017, Copyright (C 2017 American Chemical Society 
The non-equilibrium electrons that did not cross the Schottky barrier relax via electron phonon-coupling over a time scale of typically $10-50$ ps for noble metals and transfer their energy to the NP lattice. ${ }^{38,39}$ The equilibration of electron and lattice temperatures produces a temperature rise of the NPs (Fig. 4b), featuring a peak value that depends on the laser fluence and on the initial temperature of the material.

From 100 ps to $10 \mathrm{~ns}$, typically, heat is transferred to the $\mathrm{TiO}_{2}$ matrix surrounding the metallic NPs via thermal conduction. ${ }^{40}$ The temperature rise of the matrix, sketched by the reddish background in Fig. 4b, favors NP growth. In the present case of irradiation at a high repetition rate, the time interval between consecutive laser pulses is only $2 \mu \mathrm{s}$, which is too short for the material to cool to room temperature and leads to thermal accumulation. ${ }^{41}$

\section{Longer timescale: Physical and chemical mechanisms involved in the NP growth.}

The growth of Ag NPs is a diffusion-limited process that requires more time than the duration of the laser pulse. Coalescence, Ostwald ripening of metallic NPs and reduction of silver ions are the main mechanisms that control NP growth and reshaping (Fig. 4b). ${ }^{21,42}$ They occur only above a temperature threshold and their yield increases non-linearly with temperature. Therefore, when using fs laser pulses, thermal accumulation needs to be exploited in order to enable the NP growth to large sizes. The conditions leading to the NP growth, however, depend also on other parameters such as absorption, thermal conductivity, heat capacity, melting temperature of the material, and the wavelength, fluence, scanning speed (or number of pulses) and pulse length of the laser. Each of the irradiation parameters has therefore to be adapted to the desired nanocomposite film.

Among the growth mechanisms, reduction of $\mathrm{Ag}^{+}$can also be activated photocatalytically through the generation of electron-hole pairs by $\mathrm{TiO}_{2}$ when the latter is excited at a higher energy than its band gap ( $>3.2 \mathrm{eV}$ ) by two-photon absorption (Figure 4ab). ${ }^{43} \mathrm{In}$ the considered irradiation conditions, two-photon absorption by $\mathrm{TiO}_{2}$ is mainly expected in the close vicinity of Ag NPs where local electric field is greatly enhanced by optical near-fields. The electron-hole pair recombination is, however, fast (ps time scale) and this effect cannot lead on its own to the generation of large NPs, which requires diffusion and aggregation of silver atoms on longer time scales.

\section{From 2D to 3D self-organization.}

We can now infer what happens in HS and LS structures whose irradiation conditions differ only by the scan speed, i.e. the number of accumulated pulses per unit area. The nanocomposite film offers two degrees of freedom for self-organization, the $\mathrm{TiO}_{2}$ matrix whose local density changes affect the surface topography and create LIPSS, and the Ag NPs that can form patterns inside the film. Structure HS (lower number of accumulated pulses) shows that $\mathrm{TiO}_{2}$ phase changes resulting in LIPSS at the top surface occur before Ag NPs have significantly grown $(<20 \mathrm{~nm})$. According to the mechanisms described above, each laser pulse is expected to tune, for a very short time, the dielectric function of the film to a metal-like state via electron excitation, thus enabling the propagation of a surface plasmon mode that modulates the energy deposition through interference while concentrating the field intensity near the top surface (top interference pattern in Figure 1a). After few tens of ps the metal-like behavior vanishes but a temperature increase occurs. The optical interference pattern turns into a temperature pattern that causes accordingly the partial collapse of the $\mathrm{TiO}_{2}$ matrix. NP growth is slow and located specifically in the grooves where the temperature is higher. ${ }^{27}$ It requires a large number of pulses (see methods) to lead to the growth of the NP grating shown in structure HS. This structure does not exhibit any self-organized pattern in depth that could

ACS Nano, 2017, 11 (5), pp 5031-5040, DOI: 10.1021/acsnano.7b01748

Publication Date (Web): May 4, 2017, Copyright (C 2017 American Chemical Society 
attest the presence of a guided mode. However, post mortem spectral characterizations (see the last sub-section) prove that the film of structure HS can sustain a guided mode. We thus attribute the absence of any buried self-organized pattern in the film depth to a too low temperature achieved inside the film, preventing NP growth except slightly in the close vicinity of the surface grooves.

Increasing the number of pulses, and thus the thermal load, changes the balance of the different processes involved. As shown by Hou et al., ${ }^{44}$ an increase in groove depth modifies the phase of the interference pattern and shifts the maxima of light towards the protuberance between two adjacent grooves. This phase-shift stops the positive feedback associated with the plasmon mode and induces the split of LIPSS, as also observed in other works. ${ }^{45}$ This is likely what happens in structure LS where AFM profiles reported in Fig. S2a Supplementary Information show now a double structure with $500 \mathrm{~nm}$ period. When exceeding the temperature threshold above which NPs can start growing inside the film, another positive feedback loop is activated between the guided mode and the buried NP grating formation. The associated thermal gradients are responsible for distributing silver into the shape of this buried grating since they promote not only the NP growth but are also a driving force for the NP motion and organization as reported in different experiments. ${ }^{16,17,46}$ It is worth stressing that the formation of the buried NP grating is not initiated by the LIPSS formation process but by the higher temperature. The surface and guided modes propagate in perpendicular directions and Ag NPs initially grown in the LIPSS grooves cannot efficiently couple light into the guided mode.

\section{Smart visual effects.}

Here, we illustrate how such self-organized nanostructured films can offer a combination of visual effects easy to observe by eye that could lead to innovative security features. Each of the studied samples features periodic nanostructures that can diffract light. Owing to its subwavelength period, the buried NP grating of structure LS does not diffract light under normal incidence. However, one can capture bright diffractive colors under grazing back diffraction as sketched in Fig 5a. Pictures showing green, yellow and pink colors have been taken with a smartphone by changing the incidence angle of a white LED light. Note that diffraction is observed only perpendicular to the embedded NP grating and not perpendicular to the LIPSS grating on structure LS, probably due to the low LIPSS amplitude. Conversely, in structure HS diffraction occurs perpendicular to the LIPSS, in the grooves of which NPs have grown, strongly diffracting visible light (8\% diffraction efficiency at $405 \mathrm{~nm}$ under normal incidence) under the conditions sketched in the bottom part of Fig. 5a. An optical property that is more specific to this kind of nanostructured composite films is their ability to exhibit dichroic colors in specular reflection and transmission. Fig. 5b shows several laser-written nanostructures, produced by slightly varying the scanning speed and the laser power around the values used for structures LS and HS to aim at tuning the spectral response. These patterns illustrate the large color gamut provided by such a laser writing technique and show that interesting color matching can be found between different nanostructured areas when observed under specific conditions. As an example of application of such a "polarization sensitive color matching", we show a pattern made of nine squares written with three different laser irradiation conditions that displays two different geometrical forms, either a (yellow) vertical line or a (pinkish) cross, depending on the polarization of the illuminating light. This smart effect may be used as a security feature for visual authentication.

ACS Nano, 2017, 11 (5), pp 5031-5040, DOI: 10.1021/acsnano.7b01748

Publication Date (Web): May 4, 2017, Copyright (C 2017 American Chemical Society 
(a) $-1^{\text {st }}$ order diffraction

LS NP grating, $\Lambda=310 \mathrm{~nm}$
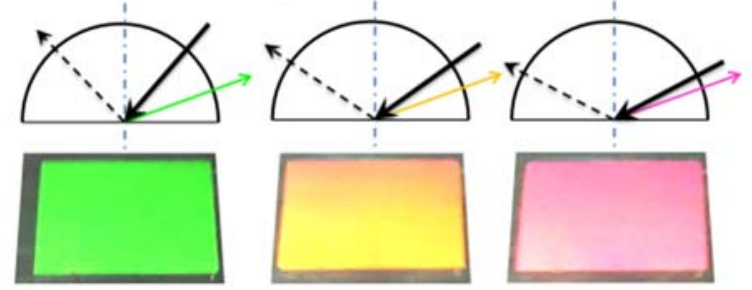

HS NP grating in LIPSS, $\Lambda=490 \mathrm{~nm}$

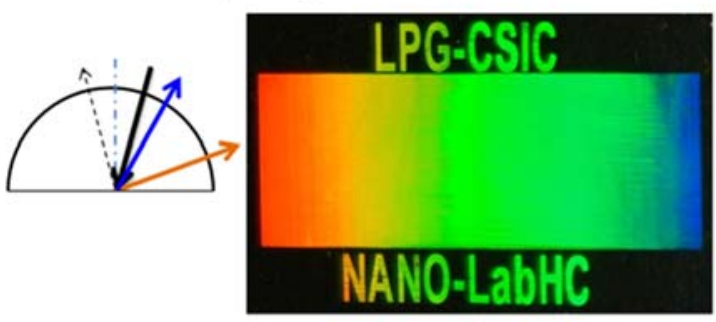

(b)

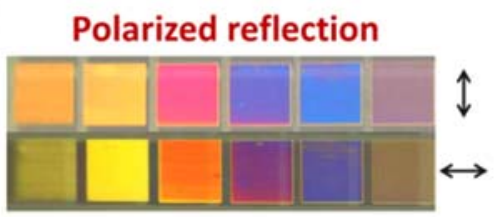

Polarized transmission

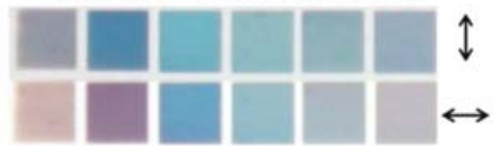

Polarization sensitive color matching

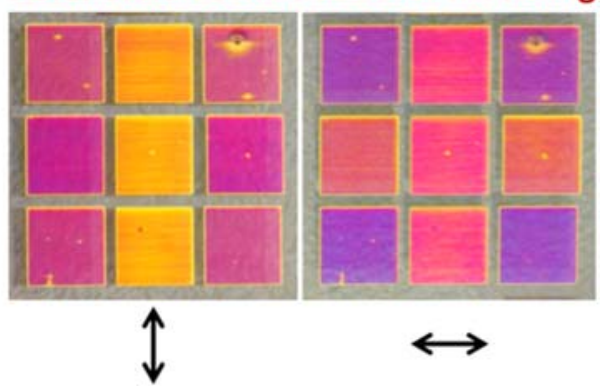

Figure 5: Smart visual effects of 2D and 3D self-organized nanocomposite films. (a) Colors observed in the $-1^{\text {st }}$ diffraction order of the buried NP grating in structures LS (NP grating perpendicular to LIPSS) and HS (NP grating in LIPSS). (b) Colors observed in reflection and transmission through a linear polarizer placed in front of the camera and oriented along the double arrow. In the "polarization sensitive color matching" image the color of the two external squares of the middle row is selected to match: the color of the four corners for the vertical polarization and the color of the middle column for the horizontal polarization. This pattern allows to display two different colored geometrical forms in reflection depending on light polarization.

\section{Origin of dichroic colors}

The polarization sensitivity of colors observed in structures LS and HS is discussed below by analyzing their reflection and transmission spectra. Transmission spectra of structure HS recorded under various incidence angles (Figure 6a-b) are polarization dependent and exhibit two characteristic dips for TE polarization (polarization parallel to the LIPSS grating). The broader one, whose minimum is around $510 \mathrm{~nm}$ is mainly due to the plasmon absorption of silver NPs and does not significantly change with the incidence angle from $0^{\circ}$ to $8^{\circ}$ (Fig. 6a). On the other hand, the smaller dip located at $790 \mathrm{~nm}$, which nearly disappears for TM polarization, splits into two dips at longer and shorter wavelengths when increasing the incidence angle. This split in two dips, whose spectral distance increases with the incidence angle, is characteristic of the excitation of quasi-guided modes propagating inside the film in directions perpendicular to the grating lines. ${ }^{19}$ Under oblique incidence such modes are coupled in the film by the grating structure in forward and backward direction for wavelengths $\lambda^{+}$and $\lambda^{-}$, respectively, given by equation: $\lambda^{ \pm}=\left[\operatorname{Re}\left(\eta^{ \pm}\right) \pm \sin \left(\theta_{\text {inc }}\right)\right] \times \Lambda$, where $\eta^{ \pm}$is the effective refractive index of the forward or backward mode and $\theta_{\text {inc }}$ the incidence angle. They are also coupled out (leaky modes) and interfere with the transmitted (or reflected) beam leading to dips in the transmission (or reflection) spectrum. ${ }^{47}$ The strong sensitivity of these resonance modes to the polarization (the coupling being inefficient for TM polarization) is one of the causes of the sample dichroism. It however does not explain the significant broadening and red-shift of the broader resonance at around 500-600 $\mathrm{nm}$ observed when rotating the polarization from TE to TM on transmission, reflection or loss spectra (Fig. 6c-d). The latter indicate that NPs might be elongated along the direction perpendicular to the LIPSS or that a

ACS Nano, 2017, 11 (5), pp 5031-5040, DOI: 10.1021/acsnano.7b01748

Publication Date (Web): May 4, 2017, Copyright (C 2017 American Chemical Society 
stronger near-field coupling between adjacent NPs might occur in this direction. Unfortunately, the analysis of the STEM images does not provide clear evidence of the presence of oriented elliptical NPs or a significantly smaller distance between NPs along the direction perpendicular to the LIPSS, which would explain a stronger near-field coupling for TM polarization. Yet, given the high sensitivity of the optical response to NP shape or distribution anisotropy, we suspect that both effects do contribute, in addition to far-field coupling.

(a)

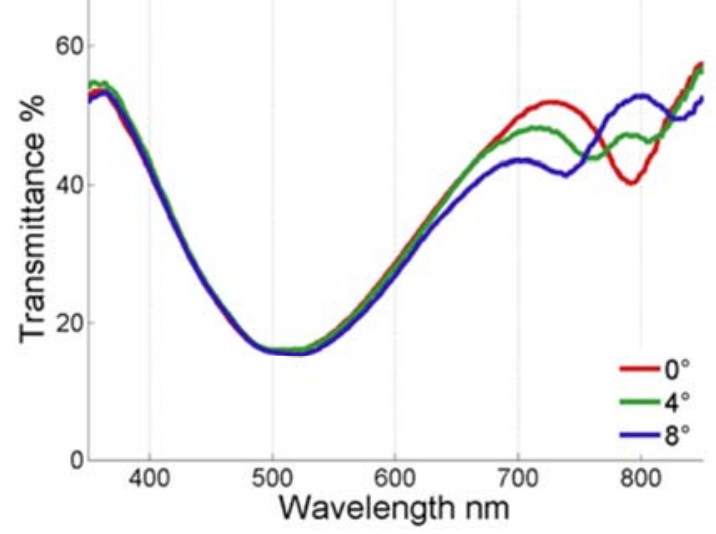

(c)

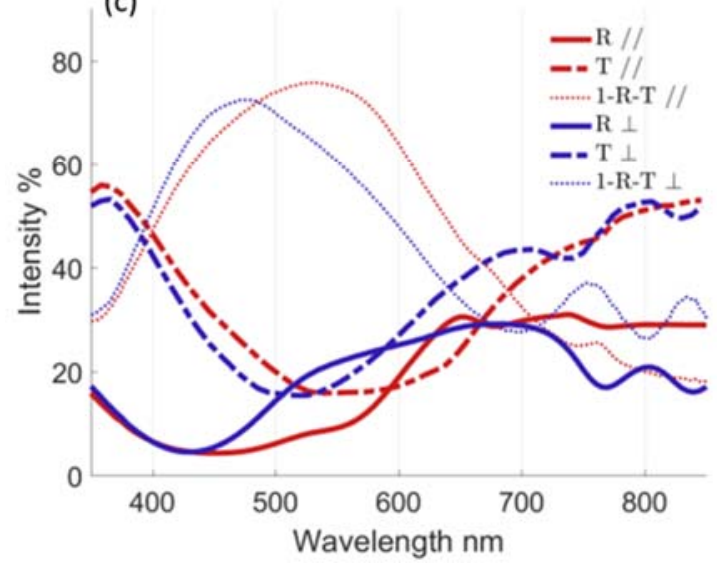

(b)

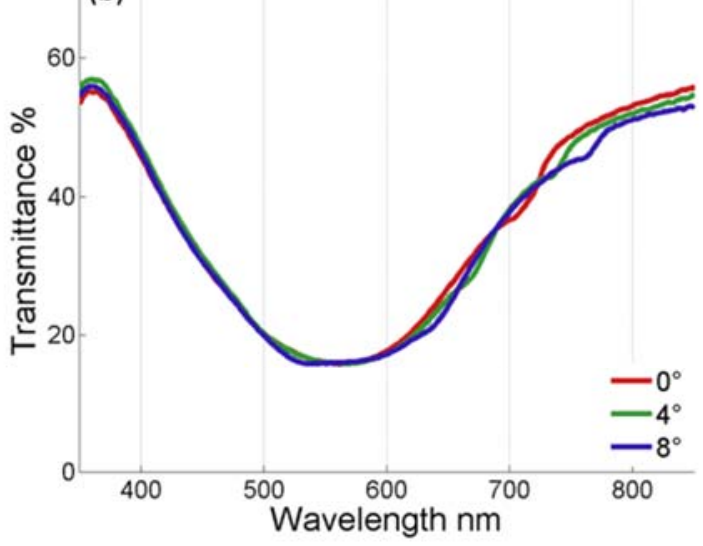

(d)

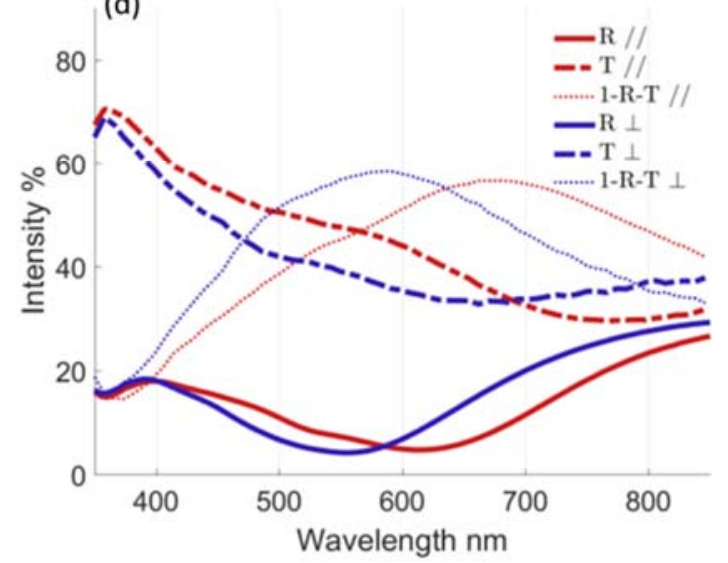

Figure 6: Spectral characterizations. Top: Transmission spectra of HS structure under different incidence angles with a probe polarization parallel (a) or perpendicular (b) to the LIPSS direction. The incidence plane is perpendicular to the LIPSS. Bottom: transmission (T) and reflection (R) spectra measured under $8^{\circ}$ of incidence for structure HS (c) and LS (d). Curves 1-R-T correspond to the spectral variations of all losses including absorption and diffraction. $\perp$ and // refer to the probe polarization relative to the laser polarization.

The spectra also allow a more detailed characterization of the guided modes and properties of processed films. The refractive index of the initial mesoporous $\mathrm{TiO}_{2}$ film with no silver has been measured as $n_{\text {film }}=1.66$ by ellipsometry and on a glass substrate of index $\mathrm{n}_{\text {substrate }}=1.5$, it can sustain guided modes for a film thickness larger than $\mathrm{d} \approx 116 \mathrm{~nm}$. The refractive index of the film is expected to increase when adding silver and when densifying the film upon laser treatment, which further enhances mode confinement. If one takes the location of the dips as the resonance wavelength, the effective index of the guided mode in structure HS is given by $\operatorname{Re}(\eta) \approx 1.61$. This value is consistent with the effective index of a mode guided in the $\mathrm{TiO}_{2}$-based film. It can be compared to the effective index of the guided mode in the LS structure, obtained by relating the fs laser wavelength to the period of the buried NP grating, $\eta=\lambda / \Lambda=515 / 310=1.66$. The higher value obtained for the LS structure is consistent with a higher refractive index caused by stronger film densification and larger NPs.

ACS Nano, 2017, 11 (5), pp 5031-5040, DOI: 10.1021/acsnano.7b01748

Publication Date (Web): May 4, 2017, Copyright (C 2017 American Chemical Society 
It is worth noting that the wavelength resonance is not expected to be exactly at the dip position in such absorbing systems, in which the spectral overlap of the LSPR and the waveguide resonance creates mode hybridization, but in a reasonable approximation.

\section{CONCLUSION}

In this paper we demonstrate that two independent self-organization processes can be triggered simultaneously in a nanocomposite film upon fs laser irradiation, leading to 3D nanostructuring of a plasmonic system. The $\mathrm{TiO}_{2}$ matrix surface, which can sustain a surface mode during the fs laser pulse thanks to its transient metallic behavior, undergoes a periodic local densification originating from an interference pattern located near the film top surface. Metallic atoms that diffuse into the film volume can independently aggregate into a selforganized buried NP grating provided that a guided mode can be excited in the film and the temperature rise is high and long enough to promote the NP growth.

This strategy is compatible with high-speed scanning for the fabrication of highly ordered metal-dielectric nanostructured films over large areas in short processing times. The technique can be extended to a nanostructuring concept based on multilayer stacks, specifically designed to sustain guided waves in specific layers and provide self-organization of NPs at pre-defined depths. Applications of such systems can be found in photovoltaic or photocatalytic devices since greatly enhancing the light interaction with $\mathrm{TiO}_{2}$. Moreover, the diffractive and polarization-sensitive properties of these plasmonic $\mathrm{Ag}: \mathrm{TiO}_{2}$ nanostructures are also of great promise for multiplexed optical image encoding and security.

\section{MATERIALS AND METHODS}

Material. The samples consist of glass slides coated with a mesoporous thin film of amorphous $\mathrm{TiO}_{2}$ impregnated with silver salt. The mesoporous titania film is elaborated by a sol-gel process. The sol contains titanium tetraisopropoxide (TTIP, Aldrich; 97\%), acetylacetone (AcAc, Aldrich; 99\%), hydrochloric acid ( $\mathrm{HCl}$, Roth; 37\%), ethanol (EtOH, Carlo Erba; absolute), Pluronic P123 ((PEO) $)_{20}(\mathrm{PPO})_{70}(\mathrm{PEO})_{20}$, Aldrich; MW: 5000$)$ and de-ionized water $\left(\mathrm{H}_{2} \mathrm{O}\right)$ with the following molar ratios: TTIP/P123/ethanol/ $/ \mathrm{HCl} / \mathrm{H}_{2} \mathrm{O} / \mathrm{AcAc}=1: 0.025: 28.5: 0.015: 29.97: 0.5$. Pluronic P123 is a symmetric triblock copolymer constituted of poly(ethylene oxide)(PEO) and poly (propylene oxide) (PPO). Films are dip-coated and calcined at $340^{\circ} \mathrm{C}$ to form mesoporous titania films. The pore size of the films ranges from $5 \mathrm{~nm}$ to $20 \mathrm{~nm}$ and their thickness is estimated to $230 \pm 50 \mathrm{~nm}$. Silver ions are introduced within the mesopority by soaking the films in an aqueous ammoniacal silver nitrate solution $(0.75 \mathrm{M})$ for $30 \mathrm{~min}$. After rinsing and drying, they are exposed to UV light $\left(400 \mu \mathrm{W} . \mathrm{cm}^{-2}\right.$ at a wavelength of $\left.254 \mathrm{~nm}\right)$ for $30 \mathrm{~min}$. This initiates the growth of a high density of small Ag NPs with typically two size distributions, as characterized in reference ${ }^{42}$ centred at $3 \mathrm{~nm}$ for nanoparticles located in the $100 \mathrm{~nm}$ upper part of the film and at $1.5 \mathrm{~nm}$ for nanoparticles located in the rest of the film.

Laser irradiation. The laser system used for irradiation was a fiber-based femtosecond laseramplifier (Tangerine, Amplitude Systems), which delivers laser pulses with a pulse duration of $\Delta \mathrm{t} \approx 370 \mathrm{fs}$ at a fundamental wavelength of $1030 \mathrm{~nm}$ and a constant repetition rate of $f_{\text {rep }}=500$ $\mathrm{kHz}$. In order to excite the LSPR of the Ag NPs the laser wavelength was frequency-doubled to $515 \mathrm{~nm}$ by means of a BBO crystal. The pulse energy was adjusted by means of a lambda-half $(\lambda 2)$ wave-plate combined with a double thin film polarizer. A second $N 2$ wave-plate was used to control the polarization state of the pulse incident on the sample, which is a critical parameter in defining the orientation of the nanogratings produced. The Gaussian laser beam passed a galvanometric mirror scanning system combined with an F-theta lens $(f=100 \mathrm{~mm}$ )

ACS Nano, 2017, 11 (5), pp 5031-5040, DOI: 10.1021/acsnano.7b01748

Publication Date (Web): May 4, 2017, Copyright @ 2017 American Chemical Society 
for scanning the focused beam over the sample surface along lines in the direction perpendicular to the laser polarization. The focused spot diameter at the sample plane was $d=$ $33 \mu \mathrm{m}\left(1 / \mathrm{e}^{2}\right.$ intensity). Experiments were performed at a variety of scan speeds within the range $1 \mathrm{~m} . \mathrm{s}^{-1}-1 \mathrm{~mm} \cdot \mathrm{s}^{-1}$ and laser fluences from 29 to $62 \mathrm{~mJ} . \mathrm{cm}^{-2}$. The scan speeds referring to samples HS and LS, which are given in the article, can actually lie in the ranges $v_{H S} \in[50-120]$ $\mathrm{mm} . \mathrm{s}^{-1}$ and $v_{L S} \in[5-40] \mathrm{mm} . \mathrm{s}^{-1}$ for laser fluence within the range $46-54 \mathrm{~mJ} . \mathrm{cm}^{-2}$. The laser fluence used for images reported in the article is $50 \mathrm{~mJ}_{\mathrm{cm}} \mathrm{cm}^{-2}$. Using the relation $N_{\text {eff, }}=d \times f_{\text {rep }} / v$, the effective pulse number ( $1 / \mathrm{e}^{2}$ intensity criterion) per line scan was $N_{\text {eff,HS }}=165$ and $N_{\text {eff, } L S}=$ 1650 , respectively. The $\mathrm{TiO}_{2}$ surface was scanned in $2 \mathrm{D}$ by using a high spatial overlap (94\%) between adjacent laser lines. This optimized scan parameters provided high uniformity as recently reported for the production of LIPSS on homogeneous materials. ${ }^{48}$

Microscopy. FIB thin lamellae were made using a focused ion beam/scanning electron microscope (FIB/SEM) FEI Helios 600i. The film was protected by carbon layers of increasing density deposited by evaporation, electron beam and ion beam to prevent charging effect and ion damage of the film. FIB/SEM process was chosen to extract a lamella from the exact middle of a laser scanned line. Rough milling, $U$ cut, transfer of the lamellae on a transmission electron microscopy (TEM) half grid, thinning was carried out. Particular attention was paid to the cleaning of the lamellae at several decreasing ion beam voltages, down to $1 \mathrm{kV}$.

STEM-EELS (electron energy loss spectroscopy) and STEM-diffraction experiments were carried out in a FEI Titan ETEM G2 80-300, operated at $300 \mathrm{kV}$, and equipped with a Gatan Imaging Filter (GIF) Tridiem 866 ER and a Gatan OneView ${ }^{\mathrm{TM}} 4 \mathrm{k}^{*} 4 \mathrm{~K}$ CCD. STEM-EELS datasets were acquired using the spectrum imaging technique, and elemental maps were postprocessed by applying principal component analysis to reduce the noise level. STEM-diffraction datasets were acquired using the 4DSTEM plugin implemented in Gatan Microscopy Suite v3, with a convergence angle of $\sim 3$ mrad. The resolution of 4D STEM analysis is defined by the effective size of the electron beam on the specimen, and was $\sim 1 \mathrm{~nm}$. Only the areas for which at least four adjacent pixels have the same crystal phase have been labeled on the reconstructed bright field image in figure $2 \mathrm{~g}$. Other darker areas are very likely to be mainly $\mathrm{Ag}$ NPs but their diffraction signal was too low to be separated from the background noise. HAADF-STEM images were acquired in a Jeol 2010F TEM operated at $200 \mathrm{kV}$. For plane-view imaging, samples were prepared by depositing on a Cu grid shavings of structures obtained by scraping the film. The SEM used is a FEI Nova nanoSEM 200 model, working in low vacuum mode and equipped with a Helix detector. Under these conditions, we were able to perform SEM experiments without need of any conductive coating. Topography measurements were carried out with an Agilent 5500 AFM in tapping mode.

\section{ASSOCIATED CONTENT}

Supporting Information

The Supporting Information is available free of charge on the ACS Publications website at DOI:

-Additional characterizations of 2D and 3D self-organized nanocomposite films.

-Discussion about the laser-induced temperature rise.

\section{AUTHOR INFORMATION}

Corresponding Author

*E-mail: nathalie.destouches@univ-st-etienne.fr.

*E-mail: j.siegel@io.cfmac.csic.es.

Nathalie Destouches: 0000-0002-3843-6382

Jan Siegel: 0000-0002-6159-7299

Notes: The authors declare no competing financial interest.

ACS Nano, 2017, 11 (5), pp 5031-5040, DOI: 10.1021/acsnano.7b01748

Publication Date (Web): May 4, 2017, Copyright (C 2017 American Chemical Society 


\section{ACKNOWLEDGEMENTS}

This work was funded by the French Research Agency (ANR) in the framework of project PHOTOFLEX 12-NANO-0006 and programs ANR-10-LABX-0075 (Labex MANUTECH-SISE) and ANR-11-IDEX-0007. It has also been supported by the LiNaBioFluid project (H2020-FETOPEN2014-2015RIA, grant 665337) of the European Commission, as well as by a research grant (TEC2014-52642-C2-1-R) from the Spanish Ministry of Economy and Competitiveness. M.G.-L. thanks the Spanish Ministry of Education for a FPU fellowship. The authors thank CLYM (www.clym.fr) for access to the Jeol 2010F TEM and the FEI Titan ETEM G2 80-300. GIE MANUTECH-USD is acknowledged for providing access to the FEI Dual Beam Helios600i.

\section{REFERENCES}

1. Kristensen, A.; Yang, J.K.; Bozhevolnyi, S.I.; Link, S.; Nordlander, P.; Halas, N.J.; Mortensen, N.A. Plasmonic Colour Generation. Nat. Rev. Mater. 2016, 2, 16088.

2. Rycenga, M.; Cobley, C.M.; Zeng, J.; Li, W.; Moran, C.H.; Zhang, Q.; Qin, D.; Xia, Y. Controlling the Synthesis and Assembly of Silver Nanostructures for Plasmonic Applications. Chem. Rev. 2011, 111, 3669-3712.

3. Zijlstra, P.; Chon, J.W.; Gu, M. Five-Dimensional Optical Recording Mediated by Surface Plasmons in Gold Nanorods. Nature 2009, 459, 410.

4. Stalmashonak, A.; Abdolvand, A.; Seifert, G. Metal-Glass Nanocomposite for Optical Storage of Information. Appl. Phys. Lett. 2011, 99, 201904.

5. Ellenbogen, T.; Seo, K.; Crozier, K.B. Chromatic Plasmonic Polarizers for Active Visible Color Filtering and Polarimetry. Nano Lett. 2012, 12, 1026-1031.

6. Le Bris, A.; Maloum, F.; Teisseire, J.; Sorin, F. Self-Organized Ordered Silver Nanoparticle Arrays Obtained by Solid State Dewetting. Appl. Phys. Lett. 2014, 105, 203102.

7. Park, C.; Yoon, J.; Thomas, E.L. Enabling Nanotechnology with Self Assembled Block Copolymer Patterns. Polymer 2003, 44, 6725-6760.

8. Fort, E.; Ricolleau, C.; Sau-Pueyo, J. Dichroic Thin Films of Silver Nanoparticle Chain Arrays on Facetted Alumina Templates. Nano Lett. 2003, 3, 65-67.

9. Sanchez-Valencia, J.R.; Toudert, J.; Borras, A.; Barranco, A.; Lahoz, R.; De la Fuente, G.F.; Frutos, F.; Gonzalez-Elipe, A.R. Selective Dichroic Patterning by Nanosecond Laser Treatment of Ag Nanostripes. Adv. Mater. 2011, 23, 848-853.

10. Chon, J.W.; Bullen, C.; Zijlstra, P.; Gu, M. Spectral Encoding on Gold Nanorods Doped in a Silica Sol-Gel Matrix and Its Application to High-Density Optical Data Storage. Adv. Func. Mater. 2007, 17, 875-880.

11. Stalmashonak, A.; Podlipensky, A.; Seifert, G.; Graener, H. Intensity-Driven, Laser induced Transformation of Ag Nanospheres to Anisotropic Shapes. Appl. Phys. B 2009, 94, 459-465.

12. Stalmashonak, A.; Matyssek, C.; Kiriyenko, O.; Hergert, W.; Graener, H.; Seifert, G. Preparing Large-Aspect-Ratio Prolate Metal Nanoparticles in Glass by Simultaneous Femtosecond Multicolor Irradiation. Opt. Lett. 2010, 35, 1671-1673.

13. Baraldi, G.; Gonzalo, J.; Solis, J.; Siegel, J. Reorganizing and Shaping of Embedded Near-Coalescence Silver Nanoparticles with Off-Resonance Femtosecond Laser Pulses. Nanotechnology 2013, 24, 255301.

ACS Nano, 2017, 11 (5), pp 5031-5040, DOI: 10.1021/acsnano.7b01748

Publication Date (Web): May 4, 2017, Copyright (C) 2017 American Chemical Society 
14. Doster, J.; Baraldi, G.; Gonzalo, J.; Solis, J.; Hernandez-Rueda, J.; Siegel, J. Tailoring the Surface Plasmon Resonance of Embedded Silver Nanoparticles by Combining Nano-and Femtosecond Laser Pulses. Appl. Phys. Lett. 2014, 104, 153106.

15. Yadavali, S.; Sachan, R.; Dyck, O.; Kalyanaraman, R. "DC Electric Field Induced Phase Array Self-Assembly of Au Nanoparticles. Nanotechnology 2014, 25, 465301.

16. Eurenius, L.; Hägglund, C.; Olsson, E.; Kasemo, B.; Chakarov, D. Grating Formation by Metal-Nanoparticle-Mediated Coupling of Light into Waveguided Modes. Nat. Photonics 2008, 2, 360-364.

17. Reinhardt, H.M.; Bücker, K.; Hampp, N.A. Directed Assembly of Gold Nanowires on Silicon via Reorganization and Simultaneous Fusion of Randomly Distributed Gold Nanoparticles. Opt. Express 2015, 23, 11965-11974.

18. Loeschner, K.; Seifert, G.; Heilmann, A. Self-Organized, Gratinglike Nanostructures in Polymer Films with Embedded Metal Nanoparticles Induced by Femtosecond Laser Irradiation. J. Appl. Phys. 2010, 108, 073114.

19. Destouches, N.; Crespo-Monteiro, N.; Vitrant, G.; Lefkir, Y.; Reynaud, S.; Epicier, T.; Liu, Z.; Vocanson, F.; Pigeon, F. Self-Organized Growth of Metallic Nanoparticles in a Thin Film under Homogeneous and Continuous-Wave Light Excitation. J. Mater. Chem. C 2014, 2, 6256-6263.

20. Baraldi, G.; Bakhti, S.; Liu, Z.; Reynaud, S.; Lefkir, Y.; Vocanson, F.; Destouches, N. Polarization-Driven Self-Organization of Silver Nanoparticles in 1D and 2D Subwavelength Gratings for Plasmonic Photocatalysis. Nanotechnology 2017, 28, 035302.

21. Liu, Z.; Vitrant, G.; Lefkir, Y.; Bakhti, S.; Destouches, N. Laser Induced Mechanisms Controlling the Size Distribution of Metallic Nanoparticles. Phys. Chem. Chem. Phys. 2016, 18, 24600-24609.

22. Birnbaum, M. Semiconductor Surface Damage Produced by Ruby Lasers. J. Appl. Phys. 1965, 36, 3688-3689.

23. Öktem, B.; Pavlov, I.; Ilday, S.; Kalaycıoğlu, H.; Rybak, A.; Yavaş, S.; Erdoğan, M.; Ilday, F.Ö. Nonlinear Laser Lithography for Indefinitely Large-Area Nanostructuring with Femtosecond Pulses. Nat. Photonics 2013, 7, 897-901.

24 Siegman, A. E.; Fauchet, P.M. Stimulated Woods Anomalies on Laser Illuminated Surfaces. IEEE J. Quant. Elect. 1986, 22, 1384-1403.

25. Bonse, J.; Krüger, J.; Höhm, S.; Rosenfeld, A. Femtosecond Laser-Induced Periodic Surface Structures. J. Laser Appl. 2012, 24, 042006.

26. Sipe, J.E.; Young, J.F.; Preston, J.S.; Van Driel, H.M. Laser-Induced Periodic Surface Structure. I. Theory. Phys. Rev. B 1983, 27, 1141.

27. Huang, M.; Zhao, F.; Cheng, Y.; Xu, N.; Xu, Z. Origin of Laser-linduced NearSubwavelength Ripples: Interference Between Surface Plasmons and Incident Laser. ACS Nano 2009, 3, 4062-4070.

28. Garrelie, F.; Colombier, J.P.; Pigeon, F.; Tonchev, S.; Faure, N.; Bounhalli, M.; Reynaud, S.; Parriaux, O. Evidence of Surface Plasmon Resonance in Ultrafast Laser-Induced Ripples. Opt. Express 2011, 19, 9035-9043.

29. Garcia-Lechuga, M.; Puerto, D.; Fuentes-Edfuf, Y.; Solis, J.; Siegel, J. Ultrafast MovingSpot Microscopy: Birth and Growth of Laser-Induced Periodic Surface Structures. ACS Photonics 2016, 3, 1961-1967.

30. Hohlfeld, J.; Müller, J.G.; Wellershoff, S.S.; Matthias, E. Time-Resolved Thermoreflectivity of Thin Gold Films and its Dependence on Film Thickness. Appl. Phys. B 1997, 64, 387390.

31 Linic, S.; Christopher, P.; Ingram, D.B. Plasmonic-Metal Nanostructures for Efficient Conversion of Solar to Chemical Energy. Nat. Mater. 2011, 10, 911-921.

ACS Nano, 2017, 11 (5), pp 5031-5040, DOI: 10.1021/acsnano.7b01748

Publication Date (Web): May 4, 2017, Copyright (C 2017 American Chemical Society 
32. Clavero, C. Plasmon-Induced Hot-Electron Generation at Nanoparticle/Metal-Oxide Interfaces for Photovoltaic and Photocatalytic Devices. Nat. Photonics 2014, 8, 95-103.

33. Tian, Y.; Tatsuma, T. Mechanisms and Applications of Plasmon-Induced Charge Separation at $\mathrm{TiO}_{2}$ Films Loaded with Gold Nanoparticles. J. Am. Chem. Soc. 2005, 127, 7632-7637.

34. Furube, A.; Du, L.; Hara, K.; Katoh, R.; Tachiya, M. Ultrafast Plasmon-Induced Electron Transfer from Gold Nanodots into $\mathrm{TiO}_{2}$ Nanoparticles. J. Am. Chem. Soc. 2007, 129, 14852-14853.

35. Bulgakova, N.M.; Stoian, R.; Rosenfeld, A.; Hertel, I.V.; Campbell, E.E.B. Electronic Transport and Consequences for Material Removal in Ultrafast Pulsed Laser Ablation of Materials. Phys. Rev. B 2004, 69, 054102.

36. Kazuma, E.; Tatsuma, T. Photoinduced Reversible Changes in Morphology of Plasmonic Ag Nanorods on $\mathrm{TiO}_{2}$ \& Application to Versatile Photochromism, Chem. Commun. 2012, 48, 1733-1735.

37. Crespo-Monteiro, N.; Destouches, N.; Nadar, L.; Reynaud, S.; Vocanson, F.; Michalon, J. $\mathrm{Y}$. Irradiance Influence on the Multicolor Photochromism of Mesoporous $\mathrm{TiO}_{2}$ Films Loaded with Silver Nanoparticles, Appl. Phys. Lett. 2011, 99, 173106.

38. Wellershoff, S.-S.; Hohlfeld, J.; Güdde, J.; Matthias, E. The Role of Electron-Phonon Coupling in Femtosecond Laser Damage of Metals, Appl. Phys. A 1999, 69, S99-S107.

39. Hartland, G. V. Optical Studies of Dynamics in Noble Metal Nanostructures, Chem. Rev. 2011, 111, 3858-3887.

40. Brongersma, M. L.; Halas, N. J.; Nordlander, P. Plasmon-Induced Hot Carrier Science \& Technology, Nat. Nanotechnol. 2015, 10, 25-34.

41. Eaton, S.M.; Zhang, H.; Ng, M.L.; Li, J.; Chen, W.J.; Ho, S.; Herman, P.R. Transition from Thermal Diffusion to Heat Accumulation in High Repetition Rate Femtosecond Laser Writing of Buried Optical Waveguides. Opt. Express 2008, 16, 9443-9458.

42. Liu, Z.; Destouches, N.; Vitrant, G.; Lefkir, Y.; Epicier, T.; Vocanson, F.; Bakhti, S.; Fang, Y.; Bandyopadhyay, B.; Ahmed, M. Understanding the Growth Mechanisms of Ag Nanoparticles Controlled by Plasmon-Induced Charge Transfers in $\mathrm{Ag}^{-\mathrm{TiO}_{2}}$ Films, J. Phys. Chem. C. 2015, 119, 9496-9505.

43. Crespo-Monteiro, N.; Destouches, N.; Bois, L.; Chassagneux, F.; Reynaud, S.; Fournel, T. Reversible and Irreversible Laser Microinscription on Silver-Containing Mesoporous Titania Films. Adv. Mater. 2010, 22, 3166-3170.

44. Hou, S.; Huo, Y.; Xiong, P.; Zhang, Y.; Zhang, S.; Jia, T.; Sun, Z.; Qiu, J.; Xu, Z. Formation of Long-\& Short-periodic Nanoripples on Stainless Steel Irradiated by Femtosecond Laser Pulses. J. Phys. D 2011, 44, 505401.

45. Liao, Y.; Pan, W.; Cui, Y.; Qiao, L.; Bellouard, Y.; Sugioka, K.; Cheng, Y. Formation of Involume Nanogratings with Sub-100-nm Periods in Glass by Femtosecond Laser Irradiation. Opt. Lett. 2015, 40, 3623-3626.

46. Habenicht, A.; Olapinski, M.; Burmeister, F.; Leiderer, P.; Boneberg, J. Jumping Nanodroplets. Science 2005, 309, 2043-2045.

47 Tikhodeev, S. G.; Yablonskii, A. L.; Muljarov, E. A.; Gippius, N. A.; Ishihara, T. Quasiguided modes Modes and Optical Properties of Photonic Crystal Slabs. Phys. Rev. B 2002, 66, 045102.

48. Ruiz de La Cruz, A.; Lahoz, R.; Siegel, J.; De La Fuente, G.F.; Solis, J. High Speed Inscription of Uniform, Large-Area Laser-Induced Periodic Surface Structures in Cr Films Using a High Repetition Rate Fs Laser. Opt. Lett. 2014, 39, 2491-2494.

ACS Nano, 2017, 11 (5), pp 5031-5040, DOI: 10.1021/acsnano.7b01748

Publication Date (Web): May 4, 2017, Copyright (C 2017 American Chemical Society 